\title{
Femoral Nerve Injury Following a Lumbar Plexus Blockade
}

\author{
İrfan Güngör ${ }^{1}$, Murat Zinnuroğlư ${ }^{2}$, Ayça Taş ${ }^{1}$, Tolga Tezer ${ }^{1}$, Mehmet Beyazova $^{2}$ \\ ${ }^{1}$ Department of Anesthesiology and Reanimation, Gazi University Faculty of Medicine, Ankara, Turkey \\ ${ }^{2}$ Department of Physical Medicine and Rehabilitation, Gazi University Faculty of Medicine, Ankara, Turkey
}

Background: Lumbar plexus blockade (LPB) combined with sciatic nerve block (SNB) is frequently used for lower extremity surgery. Perioperative nerve injury is a rarely encountered complication of peripheral nerve blocks (PNB).

Case Report: Here we report a 44-year-old male patient who developed a partial femoral nerve injury (FNI) following a LPB which was performed before the surgery of a patellar fracture. The clinical and electroneuromyographic findings of the patient were recovered almost completely within the following six months.

Conclusion: The presented case demonstrated a FNI despite the absence of any pain or paresthesia sensation, with the disappearance of motor response under $0.3 \mathrm{~mA}$ of neurostimulation in the experienced hands. (Balkan Med J 2014;31:184-6).

Key Words: Electromyography, femoral nerve, lumbosacral plexus, nerve block, nerve stimulation, peripheral nerve injuries
Lumbar plexus block (LPB) in combination with sciatic nerve block (SNB) is a good alternative technique to general and neuroaxial anaesthesia providing intraoperative anaesthesia and postoperative analgesia in lower extremity operations. Severe complications associated with LPB mainly include systemic local anaesthetic toxicity, epidural spread of the local anaesthetic, total spinal block and retroperitoneal haematoma (1). Apart from these reported complications, femoral nerve injury (FNI) following LPB is a rarely encountered but clinically important complication (2).

In this current case, we report on an FNI following a combined LPB and SNB for treatment of a patellar fracture.

\section{CASE PRESENTATION}

A 44-year-old American Society of Anaesthesiologists (ASA) male patient, $175 \mathrm{~cm}$ tall and weighing $65 \mathrm{~kg}$, was scheduled for an open reduction and internal fixation of a patellar fracture under combined LPB and SNB. A general physical examination and laboratory tests did not reveal any abnormalities. After informing the patient, the standard institutional written consent was obtained. Monitoring of cardiopulmonary parameters was performed. Midazolam (1 $\mathrm{mg})$ and fentanyl $(0.05$ $\mathrm{mg}$ ) were administered intravenously (iv) for sedation following the placement of a venous line. Afterwards, the patient was positioned in the lateral decubitus position with the side to be blocked uppermost and antisepsis was performed on the surgical area the surgical skin area. LPB was performed with the landmarks as described by Winnie et al. (3) using a $22 \mathrm{G}, 120$ $\mathrm{mm}$, short-bevelled insulated nerve block needle (Stimuplex D, Braun, Melsungen, Germany). The femoral nerve was located at a $6 \mathrm{~cm}$ depth from the skin with observation of a quadriceps muscle motor response. The nerve stimulator (Stimuplex HNS 12 , Braun, Melsungen, Germany) was initially set to $2.0 \mathrm{~mA}$ current intensity and gradually decreased to $0.3 \mathrm{~mA}$ where the muscle twitches disappeared. Afterwards, $20 \mathrm{~mL}$ of $0.375 \%$ bupivacaine and $15 \mathrm{~mL}$ of $1.5 \%$ lidocaine was injected in 5 $\mathrm{mL}$ increments with intermittent syringe aspiration. An SNB was performed using Winnie's modified approach keeping the patient in the same position and using the same technique and needle (4). Then, $20 \mathrm{~mL}$ of $0.375 \%$ bupivacaine and $10 \mathrm{~mL}$ of $1.5 \%$ lidocaine were injected in $5 \mathrm{~mL}$ increments with intermittent syringe aspiration when a foot inversion response was observed. Blocks were evaluated via pinprick test and the operation was started approximately 15 minutes after the block. Supplementary bolus doses of midazolam $(2 \mathrm{mg})$ and fentanyl $(0.05 \mathrm{mg})$ were given (iv) for intraoperative sedoanalgesia. The operation lasted for two hours without any problem. No motor or sensory problems were observed in the physical assessment 24 hours postoperatively. The patient was discharged on the postoperative day (POD-3) with recommendations for strict bed rest and called for a control visit three weeks later. The patient had complained of weakness in his left leg at the visit 
on POD-22. The left knee extension strength was evaluated as $3 / 5$ and a significant atrophy was noted in the left quadriceps muscle. Computerized tomography (CT) of the lumbar region was carried out, which ruled out a possible haematoma in the psoas muscle.

An electrophysiological evaluation revealed findings compatible with a partial lesion of the proximal femoral nerve. The compound muscle and nerve action potentials were found to be diminished in both a motor nerve conduction study of the femoral nerve and a sensory nerve conduction study of the saphenous nerve on the left leg. Electromyography (EMG) disclosed denervation potentials accompanied by mild and significant reductions in the recruitment patterns of the left iliopsoas and vastus lateralis muscles, respectively.

A comprehensive rehabilitation programme primarily focused on strengthening the lower extremity muscles was initiated. Physical assessment in the third week of the patient's rehabilitation programme revealed normal muscle strength of the left lower extremity muscles except for the quadriceps, which was graded as $4 / 5$. The atrophy in the knee extensors had improved and a control electroneuromyographic evaluation after six months revealed no denervation potentials, normal EMG findings at the left vastus lateralis and only a minimally reduced recruitment pattern of the left vastus medialis muscle.

\section{DISCUSSION}

Perioperative nerve injury is a rarely encountered complication of peripheral nerve blocks (PNB) and may present with temporary paresthesia, hypoesthesia and muscle weakness, or rarely as permanent paresis. The aetiology of nerve injuries that develop after operations performed under PNB includes direct needle trauma, intraneural injection of local anaesthetic agents, ischaemic nerve injury secondary to compression of a haematoma following vascular injury or use of additive vasoconstrictive drugs, surgical or intraoperative positional trauma, tourniquet injury or local anaesthetic neurotoxicity (5).

The cases presented by Kaufman et al. (6) indicate a direct relationship between nerve injury and paresthesia as all the seven patients who described severe paresthesia during the procedure developed chronic pain afterwards. It is accepted that nerve stimulation reduces the risk of direct needle trauma and intraneural injection as it informs the practitioner through the evoked motor responses before the needle contacts the nerve (7). In clinical practice of PNB where ultrasonography is not used, the most important indicators suggestive of intraneural injections are the practioner's feeling of high injection pressure during local anaesthetic injection and the sensation of pain and paresthesia experienced by the patient $(6,7)$. It is recommended to avoid local anaesthetic injection when muscle twitches are seen under $0.2 \mathrm{~mA}$ current intensity and/ or high injection pressures are felt by the practioner to prevent intraneural injection and subsequent nerve injury during neurostimulation-guided PNB (8).

In this case, although the LPB was performed in line with the recommendations in the literature, the local anaesthetic was delivered without any obvious injection pressure and the patient reported no signs of paresthesia suggesting a needlenerve contact during the procedure, the development of FNI during this procedure cannot be denied. In an experimental animal study it has been shown with ultrasonography that neurostimulation may not prevent intraneural injection even if the injection is performed at the widely accepted limits of 0.2-0.5 mA (9). Moayeri et al. (10) investigated the neural architecture of the sciatic nerve comparing the cross-sectional areas from proximal to distal regions and demonstrated that the ratio of neural to non-neural tissue decreased distally. This observation was correlated by findings that show a higher vulnerability to neurological sequelae after inadvertent intraneural intrafascicular injection in the proximal parts of the sciatic nerve. In this case, an inadvertent intraneural intrafascicular injection may have occurred since we used the most proximal approach to block the femoral nerve and the ratio of fascicles to the connective tissue is higher in this region. In this case, the lack of pressure value during the injection of the drug may be considered one of the limitations. However, there was no sign of increased pressure that would suggest an intraneural injection and the anaesthesiologist performing the injection had had experience over 10 years in peripheral nerve blocks.

In conclusion, we presume that FNI may develop even in the absence of pain or paresthesia in the hands of experienced professionals and with a motor response disappearing at 0.3 $\mathrm{mA}$ current intensity. A close follow-up and early initiation of the rehabilitation may help to provide better outcomes during the recovery period.

Ethics Committee Approval: N/A.

Informed Consent: Written informed consent was obtained from patient who participated in this study.

Peer-review: Externally peer-reviewed.

Author contributions: Concept - I.G., M.Z., A.T.; Design - I.G., M.Z.,T.T.; Supervision - M.B., T.T., M.Z.; Resource - M.B., I.G., M.Z.; Materials - I.G., M.Z., A.T.; Data Collection\&/or Processing - I.G., M.Z., A.T.; Analysis\&/or Interpretation - I.G., M.Z., A.T.; Literature Search - T.T., M.B., A.T.; Writing - I.G., M.Z., T.T.; Critical Reviews - I.G., M.Z., M.B.

Conflict of Interest: No conflict of interest was declared by the authors.

Financial Disclosure: The authors declared that this study has received no financial support. 


\section{REFERENCES}

1. Touray ST, de Leeuw MA, Zuurmond WW, Perez RS. Psoas compartment block for lower extremity surgery: a meta-analysis. Br J Anaesth 2008;101:750-60. [CrossRef]

2. Brull R, McCartney CJ, Chan VW, El-Beheiry H. Neurological complications after regional anesthesia: contemporary estimates of risk. Anesth Analg 2007;10:965-74. [CrossRef]

3. Winnie AP, Ramamurthy S, Durrani Z. Plexus blocks for lower extremity surgery. Anesthesiology Review 1974;1:11-6.

4. Winnie AP. Regional anesthesia. Surg Clin North Am 1975; 55: 861-92.

5. Al-Nasser B, Palacios JL. Femoral nerve injury complicating continuous psoas compartment block. Reg Anesth Pain Med 2004;29:361-3. [CrossRef]
6. Kaufman BR, Nystrom E, Nath S, Foucher G, Nystrom A. Debilitating chronic pain syndromes after presumed intraneural injections. Pain 2000;85:283-6. [CrossRef]

7. Sala Blanch X, López AM, Carazo J, Hadzic A, Carrera A, Pomés J, et al. Intraneural injection during nerve stimulator-guided sciatic nerve block at the popliteal fossa. Br J Anaesth 2009;102:855-61. [CrossRef]

8. Borgeat A, Blumenthal S, Hadzic A. Mechanisms of neurologic complications with peripheral nerve blocks. In: Finucane BT, editor. Complications of regional anesthesia. New York: Springer; 2007:74-86. [CrossRef]

9. Chan VW, Brull R, McCartney CJ, Xu D, Abbas S, Shannon P. An ultrasonographic and histological study of intraneural injection and electrical stimulation in pigs. Anesth Analg 2007;104:1281-4. [CrossRef]

10. Moayeri N, Bigeleisen PE, Groe GJ. Quantitative architecture of the brachial plexus and surrounding compartments, and their possible significance for plexus blocks. Anesthesiology 2008;108:299-304. [CrossRef] 